\title{
Upper bounds for the rainbow connection numbers of line graphs*
}

\author{
Xueliang Li, Yuefang Sun \\ Center for Combinatorics and LPMC-TJKLC \\ Nankai University, Tianjin 300071, P.R. China \\ E-mail: lxl@nankai.edu.cn; syf@cfc.nankai.edu.cn
}

\begin{abstract}
A path in an edge-colored graph $G$, where adjacent edges may be colored the same, is called a rainbow path if no two edges of it are colored the same. A nontrivial connected graph $G$ is rainbow connected if for any two vertices of $G$ there is a rainbow path connecting them. The rainbow connection number of $G$, denoted by $\operatorname{rc}(G)$, is defined as the smallest number of colors by using which there is a coloring such that $G$ is rainbow connected. In this paper, we mainly study the rainbow connection number of the line graph of a graph which contains triangles and get two sharp upper bounds for $\operatorname{rc}(L(G))$, in terms of the number of edge-disjoint triangles of $G$ where $L(G)$ is the line graph of $G$. We also give results on the iterated line graphs.
\end{abstract}

Keywords: rainbow path, rainbow connection number, (iterated) line graph, edge-disjoint triangles.

AMS Subject Classification 2000: 05C15, 05C40

\section{Introduction}

All graphs in this paper are simple, finite and undirected. Let $G$ be a nontrivial connected graph with an edge coloring $c: E(G) \rightarrow\{1,2, \cdots, k\}, k \in \mathbb{N}$, where adjacent edges may be colored the same. A path of $G$ is called rainbow if no two edges of it are colored the same. An edge-colored graph $G$ is rainbow connected if for any two vertices there is a rainbow path connecting them. Clearly, if a graph is rainbow connected, it must be connected. Conversely, any connected graph has a trivial edge coloring that makes it rainbow connected, i.e., the coloring such that each edge has a distinct color. Thus, we define the rainbow connection number

*Supported by NSFC, PCSIRT and the " 973 " program. 
of a connected graph $G$, denoted by $\operatorname{rc}(G)$, as the smallest number of colors for which there is an edge coloring of $G$ such that $G$ is rainbow connected. An easy observation is that if $G$ has $n$ vertices then $\operatorname{rc}(G) \leq n-1$, since one may color the edges of a spanning tree with distinct colors, and color the remaining edges with one of the colors already used. Generally, if $G_{1}$ is a connected spanning subgraph of $G$, then $r c(G) \leq r c\left(G_{1}\right)$. We notice the trivial fact that $r c(G)=1$ if and only if $G$ is complete, and the fact that $r c(G)=n-1$ if and only if $G$ is a tree, as well as the easy observation that a cycle with $k>3$ vertices has rainbow connection number $\left\lceil\frac{k}{2}\right\rceil([2])$. Since a Hamiltonian graph $G$ has a Hamiltonian cycle which contains all $n$ vertices, then $G$ has rainbow connection number at most $\left\lceil\frac{n}{2}\right\rceil$. Also notice that, clearly, $\operatorname{rc}(G) \geq \operatorname{diam}(G)$ where $\operatorname{diam}(G)$ denotes the diameter of $G$.

Chartrand et al. in [2] determined that the rainbow connection numbers of some graphs including trees, cycles, wheels, complete bipartite graphs and complete multipartite graphs. Caro et al. 3] gave some results on general graphs in terms of some graph parameters, such as the order or the minimum degree of a graph. They observed that $\operatorname{rc}(G)$ can be bounded by a function of $\delta(G)$, the minimum degree of $G$. They proved that if $\delta(G) \geq 3$ then $\operatorname{rc}(G) \leq \alpha n$ where $\alpha<1$ is a constant and $n=|V(G)|$. They conjectured that $\alpha=3 / 4$ suffices and proved that $\alpha<5 / 6$. Specifically, it was proved in 3] that if $\delta=\delta(G)$ then $\operatorname{rc}(G) \leq$ $\min \left\{\frac{\ln \delta}{\delta} n\left(1+o_{\delta}(1)\right), n \frac{4 \ln \delta+3}{\delta}\right\}$. Some special graph classes, such as line graphs, have many special properties, and by these properties, we can get some interesting results on their rainbow connection numbers in terms of some graph parameters. For example, in [3] the authors got a very good upper bound for the rainbow connection number of a 2-connected graph according to their ear-decomposition. And in [6], we studied the rainbow connection numbers of line graphs of triangle-free graphs in the light of particular properties of line graphs of triangle-free graphs shown in [4], and particularly, of 2-connected triangle-free graphs according to their ear decompositions. However, we did not get bounds of the rainbow connection numbers for line graphs that do contain triangles. In this paper, we aim to investigate the remaining case, i.e., line graphs that do contain triangles, and give two sharp upper bounds in terms of the number of edge-disjoint triangles.

We use $V(G), E(G)$ for the sets of vertices and edges of $G$, respectively. For any subset $X$ of $V(G)$, let $G[X]$ denote the subgraph induced by $X$, and $E[X]$ the edge set of $G[X]$; similarly, for any subset $E_{1}$ of $E(G)$, let $G\left[E_{1}\right]$ denote the subgraph induced by $E_{1}$. Let $\mathcal{G}$ be a set of graphs, then $V(\mathcal{G})=\bigcup_{G \in \mathcal{G}} V(G)$, $E(\mathcal{G})=\bigcup_{G \in \mathcal{G}} E(G)$. We define a clique in a graph $G$ to be a complete subgraph of $G$, and a maximal clique is a clique that is not contained in any larger clique. The clique graph $K(G)$ of $G$ is the intersection graph of the maximal cliques of $G$-that is, the vertices of $K(G)$ correspond to the maximal cliques of $G$, and two of these vertices are joined by an edge if and only if the corresponding maximal cliques intersect. Let $[n]=\{1, \cdots, n\}$ denote the set of the first $n$ natural numbers. For a set $S,|S|$ denotes the cardinality of $S$. We follow the notations and terminology of [1] for those not defined here. 


\section{Some basic observations}

We first list two observations which were given in [6] and will be used in the sequel.

Observation 2.1 ([G]) If $G$ is a connected graph and $\left\{E_{i}\right\}_{i \in[t]}$ is a partition of the edge set of $G$ into connected subgraphs $G_{i}=G\left[E_{i}\right]$ and $\operatorname{rc}\left(G_{i}\right)=c_{i}$, then

$$
r c(G) \leq \sum_{i=1}^{t} c_{i} .
$$

Let $G$ be a connected graph, and $X$ a proper subset of $V(G)$. To shrink $X$ is to delete all the edges between vertices of $X$ and then identify the vertices of $X$ into a single vertex, namely $w$. We denote the resulting graph by $G / X$.

Observation 2.2 ([6]) Let $G^{\prime}$ and $G$ be two connected graphs, where $G^{\prime}$ is obtained from $G$ by shrinking a proper subset $X$ of $V(G)$, that is, $G^{\prime}=G / X$, such that any two vertices of $X$ have no common adjacent vertex in $V \backslash X$. Then

$$
r c\left(G^{\prime}\right) \leq r c(G)
$$

Now we introduce two graph operations and two corresponding results which will be used later.

Operation 1: As shown in Figure 2.1, for any edge $e=u v \in G$ with $\min \left\{\operatorname{deg}_{G}(u)\right.$, $\left.\operatorname{deg}_{G}(v)\right\} \geq 2$, we first subdivide $e$, then replace the new vertex with two new vertices $u_{e}, v_{e}$ with $\operatorname{deg}_{G^{\prime}}\left(u_{e}\right)=\operatorname{deg}_{G^{\prime}}\left(v_{e}\right)=1$ where $G^{\prime}$ is the new graph.

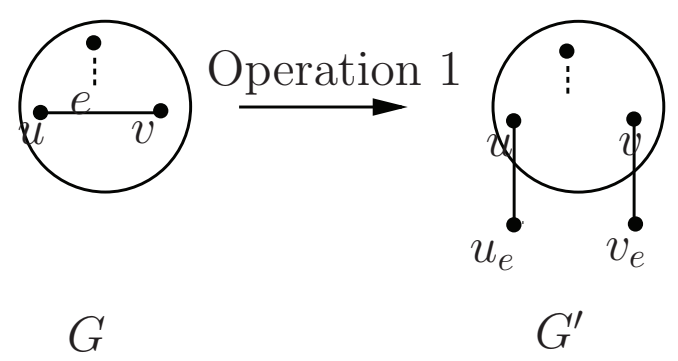

Figure $2.1 G^{\prime}$ is obtained from $G$ by doing Operation 1 to edge $e$.

Since $\operatorname{deg}_{G^{\prime}}\left(u_{e}\right)=\operatorname{deg}_{G^{\prime}}\left(v_{e}\right)=1$ in $G^{\prime}$, and by the definition of a line graph, it is easy to show that $L(G)$ can be obtained from $L\left(G^{\prime}\right)$ by shrinking a vertex set of two 
nonadjacent vertices (these two vertices correspond to edges $u u_{e}, v v_{e}$, and belong to cliques $\langle S(u)\rangle,\langle S(v)\rangle$, respectively, in $\left.L\left(G^{\prime}\right)\right)$. Recall that the line graph of a graph $G$ is the graph $L(G)$ whose vertex set $V(L(G))=E(G)$ and two vertices $e_{1}, e_{2}$ of $L(G)$ are adjacent if and only if they are adjacent in $G$. So by Observation 2.2, we have

Observation 2.3 If graph $G^{\prime}$ is obtained from a connected graph $G$ by doing Operation 1 at some edge $e \in G$, then

$$
r c(L(G)) \leq r c\left(L\left(G^{\prime}\right)\right)
$$

Operation 2. As shown in Figure 2.2, $v$ is a common vertex of a set of edge-disjoint triangles in $G$. We replace $v$ by two nonadjacent vertices $v^{\prime}$ and $v^{\prime \prime}$ such that $v^{\prime}$ is the common vertex of some triangles, and $v^{\prime \prime}$ is the common vertex of the rest triangles.

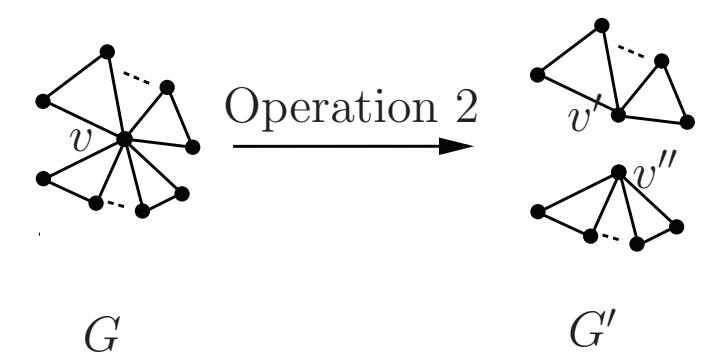

Figure 2.2 Figure of Operation 2.

Since during this procedure, the number of edges does not change, the order of the line graph $L(G)$ is equal to that of $L\left(G^{\prime}\right)$. Furthermore, by the definition of a line graph, $L\left(G^{\prime}\right)$ is a spanning subgraph of $L(G)$. So we have

Observation 2.4 If a connected graph $G^{\prime}$ is obtained from a connected graph $G$ by doing Operation 2 at some vertex $v \in G$, then

$$
r c(L(G)) \leq r c\left(L\left(G^{\prime}\right)\right)
$$

\section{Main results}

\subsection{A sharp upper bound}

Recall that the star, $S(v)$, at a vertex $v$ of graph $G$, is the set of all edges incident to $v$. A clique decomposition of $G$ is a collection $\mathscr{C}$ of cliques such that each edge of $G$ occurs in exactly one clique in $\mathscr{C}$. 
We now introduce a new terminology. For a connected graph $G$, we call $G$ a clique-tree-structure, if it satisfies the following condition:

$T_{1}$. Each block is a maximal clique.

We call a graph $H$ a clique-forest-structure, if $H$ is a disjoint union of some clique-tree-structures, that is, each component of a clique-forest-structure is a cliquetree-structure. By condition $T_{1}$, we know that any two maximal cliques of $G$ have at most one common vertex. Furthermore, $G$ is formed by its maximal cliques. The size of the clique-tree(forest)-structure is the number of its maximal cliques. An example of clique-forest-structure is shown in Figure 3.1 .
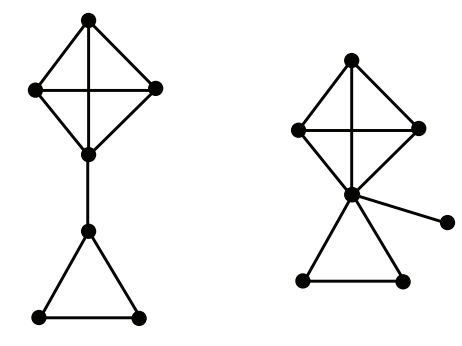

Figure 3.1 A clique-forest-structure with size 6 and 2 components.

If each block of a clique-tree-structure is a triangle, we call it a triangle-treestructure. Let $\ell$ be the size of a triangle-tree-structure. Then, by definition, it is easy to show that there are $2 \ell+1$ vertices in it. Similarly, we can give the definition of triangle-forest-structure. A clique-tree-structure $G$ is called a cliquepath-structure if the clique graph $K(G)$ is a path.

For a connected graph $G$, we call $G$ a clique-cycle-structure, if it satisfies the following three conditions:

$C_{1} \cdot G$ has at least three maximal cliques;

$C_{2}$. Each edge belongs to exactly one maximal clique;

$C_{3}$. The clique graph is a cycle. (In particular, if each maximal clique is a triangle, then it is a triangle-cycle-structure. An example of triangle-cycle-structure is shown in Figure 3.2.)

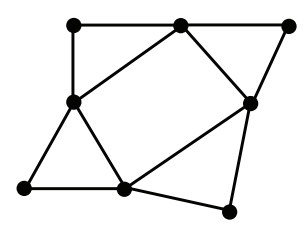

Figure 3.2 An example of triangle-cycle-structure.

An inner vertex of a graph is a vertex with degree at least two. For a graph $G$, 
we use $\overline{V_{2}}$ to denote the set of all inner vertices of $G$. Let $n_{1}=\left|\left\{v: \operatorname{deg}_{G}(v)=1\right\}\right|$, $n_{2}=\left|\overline{V_{2}}\right| .\langle S(v)\rangle$ is the subgraph of $L(G)$ induced by $S(v)$, clearly it is a clique of $L(G)$. Let $\mathscr{K}_{0}=\{\langle S(v)\rangle: v \in V(G)\}, \mathscr{K}=\left\{\langle S(v)\rangle: v \in \overline{V_{2}}\right\}$. It is easy to show that $\mathscr{K}_{0}$ is a clique decomposition of $L(G)([5])$ and each vertex of the line graph belongs to at most two elements of $\mathscr{K}_{0}$. We know that each element $\langle S(v)\rangle$ of $\mathscr{K}_{0} \backslash \mathscr{K}$, a single vertex of $L(G)$, is contained in the clique induced by $u$ that is adjacent to $v$ in $G$. So $\mathscr{K}$ is a clique decomposition of $L(G)$.

Let $X$ and $Y$ be sets of vertices of a graph $G$. We denote by $E[X, Y]$ the set of the edges of $G$ with one end in $X$ and the other end in $Y$. If $Y=X$, we simply write $E(X)$ for $E[X, X]$. When $Y=V \backslash X$, the set $E[X, Y]$ is called the edge cut of $G$ associated with $X$, and is denoted by $\partial(X)$.

Theorem 3.1 For any set $\mathcal{T}$ of $t$ edge-disjoint triangles of a connected graph $G$, if the subgraph induced by the edge set $E(\mathcal{T})$ is a triangle-forest-structure, then

$$
\operatorname{rc}(L(G)) \leq n_{2}-t .
$$

Moreover, the bound is sharp.

Proof. Let $\mathcal{T}=\bigcup_{i=1}^{c} \mathcal{T}_{i}=\bigcup_{i=1}^{c}\left\{T_{i, j_{i}}\right.$ is a triangle of $\left.\mathrm{G}: 1 \leq j_{i} \leq t_{i}\right\}\left(\sum_{i=1}^{c} t_{i}=t\right)$ be a set of $t$ edge-disjoint triangles of $G$ such that the subgraph of $G, G\left[E\left(\mathcal{T}_{i}\right)\right]$, induced by each $E\left(\mathcal{T}_{i}\right)$ is a component of the subgraph $G[E(\mathcal{T})]$, that is, a triangletree-structure of size $t_{i}$.

In $G$, for each $1 \leq i \leq c$, let $G_{i}=G\left[E\left(\mathcal{T}_{i}\right)\right], V_{i}=V\left(G_{i}\right), E_{i}=E\left(\mathcal{T}_{i}\right) ; E_{i}^{0}=$ $E\left(V_{i}\right) \cup \partial\left(V_{i}\right) \supseteq E_{i}$, and $G_{i}^{0}=G\left[E_{i}^{0}\right]$. We obtain a new graph $G^{\prime}$ from $G$ by doing Operation 1 at each edge $e \in E\left(V_{i}\right) \backslash E_{i}$ for $1 \leq i \leq c$, and we denote by $G_{i}^{\prime}$ the new subgraph (of $G^{\prime}$ ) corresponding to $G_{i}^{0}$. Applying Observation 2.3 repeatedly, we have $\operatorname{rc}(L(G)) \leq r c\left(L\left(G^{\prime}\right)\right)$.

Next we will show $\operatorname{rc}\left(L\left(G^{\prime}\right)\right) \leq n_{2}-t$. By previous discussion, we know that

$$
\mathscr{K}=\left\{\langle S(v)\rangle: v \in \overline{V_{2}}\right\}=\bigcup_{i=1}^{c}\left\{\langle S(v)\rangle: v \in V_{i}\right\} \bigcup\left\{\langle S(v)\rangle: v \in \overline{V_{2}} \backslash \bigcup_{i=1}^{c} V_{i}\right\}
$$

is a clique partition of $L(G)$. So

$$
\left\{E(\langle S(v)\rangle): v \in V_{i}\right\}_{i=1}^{c} \bigcup\left\{E(\langle S(v)\rangle): v \in \overline{V_{2}} \backslash \bigcup_{i=1}^{c} V_{i}\right\},
$$

that is,

$$
\left\{E\left(L\left(G_{i}^{0}\right)\right)\right\}_{i=1}^{c} \bigcup\left\{E(\langle S(v)\rangle): v \in \overline{V_{2}} \backslash \bigcup_{i=1}^{c} V_{i}\right\}
$$

is an edge partition of $L(G)$. So

$$
\left\{E\left(L\left(G_{i}^{\prime}\right)\right)\right\}_{i=1}^{c} \bigcup\left\{E(\langle S(v)\rangle): v \in \overline{V_{2}} \backslash \bigcup_{i=1}^{c} V_{i}\right\}
$$


is an edge partition of $L\left(G^{\prime}\right)$. By Observation 2.1, we have

$$
r c\left(L\left(G^{\prime}\right)\right) \leq \sum_{i=1}^{c} r c\left(L\left(G_{i}^{\prime}\right)\right)+\sum_{v \in \overline{V_{2}} \backslash \bigcup_{i=1}^{c} V_{i}} r c(\langle S(v)\rangle) .
$$

We know $\left|V_{i}\right|=2 t_{i}+1$, since the triangle-tree-structure $G_{i}$ has size $t_{i}$. So

$$
r c\left(L\left(G^{\prime}\right)\right) \leq \sum_{i=1}^{c} r c\left(L\left(G_{i}^{\prime}\right)\right)+\left(n_{2}-2 t-c\right) .
$$

In order to get $\operatorname{rc}\left(L\left(G^{\prime}\right)\right) \leq n_{2}-t$, we need to show $\operatorname{rc}\left(L\left(G_{i}^{\prime}\right)\right) \leq t_{i}+1$.

Claim. $r c\left(L\left(G_{i}^{\prime}\right)\right) \leq t_{i}+1$.

Proof of the Claim. Since the graph $G_{i}^{\prime}$ is obtained from $G_{i}$ by doing Operation 1 at each edge $e \in E\left(V_{i}\right) \backslash E_{i}, G_{i}^{\prime}$ contains exactly $t_{i}$ triangles: $\left\{T_{i, j_{i}} \in \mathcal{T}_{i}: 1 \leq j_{i} \leq\right.$ $\left.t_{i}\right\}$. We will show that there is a $\left(t_{i}+1\right)$-rainbow coloring of $L\left(G_{i}^{\prime}\right)$ by induction on $t_{i}$. For $t_{i}=1, G_{i}^{\prime}$ contains exactly one triangle, and we give its line graph a 2-rainbow coloring as shown in Figure 3.3. We give color 1 to the edges of $\langle S(u)\rangle$ incident with vertex $e_{1}$, edges of $\langle S(v)\rangle$ incident with vertex $e_{2}$, and edges of $\langle S(w)\rangle$ incident with vertex $e_{3}$; We then give color 2 to the edges of $\langle S(u)\rangle$ incident with vertex $e_{3}$, edges of $\langle S(v)\rangle$ incident with vertex $e_{1}$, and edges of $\langle S(w)\rangle$ incident with vertex $e_{2}$; Finally, give color 2 to the rest of the edges. It is easy to show that this is a rainbow coloring. So, the above conclusion holds for the case $t_{i}=1$. We assume

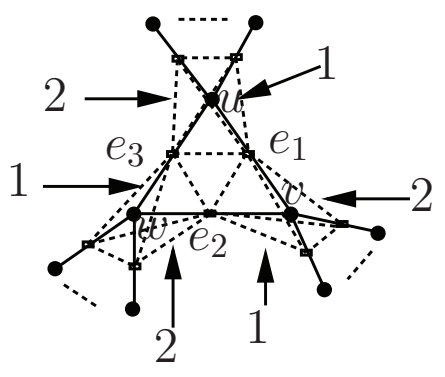

Figure 3.3 2-rainbow coloring of line graph of graph with exactly one triangle.

that the conclusion holds for the case $t_{i}=h-1$, and now show that it holds for the case $t_{i}=h$. By the definition of triangle-tree-structure, there must exist one triangle: $T=\{u, v, w\}$, which has exactly one common vertex, namely $u$, with other triangles and $v, w$ do not belong to any other triangle. We now obtain a new graph $\overline{G_{i}^{\prime}}$ from $G_{i}^{\prime}$ by doing Operation 1 at edges $e_{1}$ and $e_{2}$. It is clear that $L\left(\overline{G_{i}^{\prime}}\right)$ can be obtained from $L\left(G_{i}^{\prime}\right)$ by subdividing vertex $e_{1}$ into two vertices $\left\{e_{1}^{\prime}, e_{1}^{\prime \prime}\right\}$ and $e_{2}$ into two vertices $\left\{e_{2}^{\prime}, e_{2}^{\prime \prime}\right\}$. Since $H_{1}$ has $h-1$ (edge-disjoint) triangles, by induction hypothesis, $r c\left(L\left(H_{1}\right)\right) \leq h$. So the subgraph $L\left(G_{i}^{\prime}\right) \backslash\{\langle S(v)\rangle,\langle S(w)\rangle\} \cong L\left(H_{1}\right)$ has a rainbow $h$-coloring, and we now color the edges of $\langle S(v)\rangle$ and $\langle S(w)\rangle$ in the graph $L\left(G_{i}^{\prime}\right)$ as follows: give a new color to the edges of $\langle S(w)\rangle$ incident with vertex $e_{1}$, and 
edges of $\langle S(v)\rangle$ incident with vertex $e_{2}$. Let $e_{3}=v w$, we then give any one color, say $c_{1}$, of the former $h$ colors to the edges of $\langle S(w)\rangle$ incident with vertex $e_{3}$, and give a distinct color, say $c_{2}$, of the former $h$ colors to the edges of $\langle S(v)\rangle$ incident with vertex $e_{3}$. It is easy to show that, with above coloring, $L\left(G_{i}^{\prime}\right)$ is rainbow connected, and so $L\left(G_{i}^{\prime}\right) \leq t_{i}+1$ holds for $t_{i}=h$.

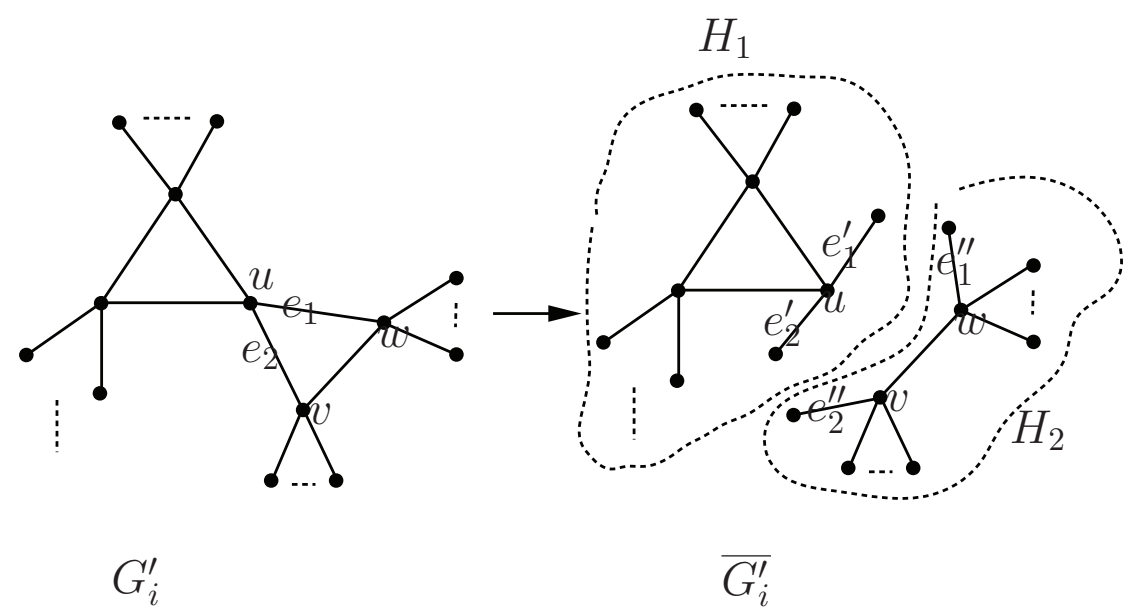

Figure $3.4 \overline{G_{i}^{\prime}}$ is obtained from $G_{i}$ by doing Operation 1 to edges $e_{1}$ and $e_{2}$.

For the sharpness of the upper bound, see Example 3.3.

We call a set of triangles independent if any two of them are vertex-disjoint. Since each single triangle is a triangle-tree-structure, applying Theorem 3.1, we have the following corollary, and for the sharpness of the upper bound, see Example 3.3.

Corollary 3.2 If $G$ is a connected graph with $t^{\prime}$ independent triangles, then

$$
r c(L(G)) \leq n_{2}-t^{\prime}
$$

Moreover, the bound is sharp.

Example 3.3 As shown in Figure 3.5, G consists of $t$ (independent) triangles and $t-1$ edges which do not belong to any triangles. Since $G$ has $3 t$ inner vertices, by Theorem 3.1(Corollary 3.2), we know $\operatorname{rc}(L(G)) \leq 2 t$; on the other hand, it is easy to show that the diameter of the line graph $L(G)$ is $2 t$, and so we have $\operatorname{rc}(L(G))=2 t$.

\subsection{Another upper bound from Theorem 3.1}

Now we use the notation similar to that of Theorem 3.1. Let $\mathcal{T}=\bigcup_{i=1}^{c} \mathcal{T}_{i}=$ $\bigcup_{i=1}^{c}\left\{T_{i, j_{i}}\right.$ is a triangle of $\left.\mathrm{G}: 1 \leq j_{i} \leq t_{i}\right\}\left(\sum_{i=1}^{c} t_{i}=t\right)$ be a set of $t$ edge-disjoint triangles of $G$; the subgraph of $G, G\left[E\left(\mathcal{T}_{i}\right)\right]$, induced by each $E\left(\mathcal{T}_{i}\right)$ is a connected 


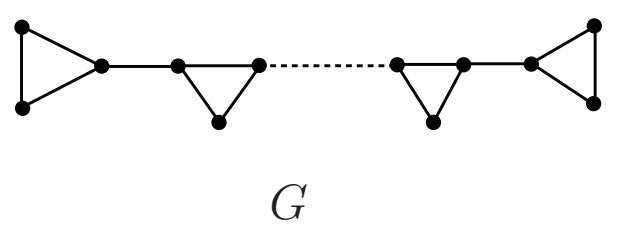

Figure 3.5 Figure of Example 3.3.

component of the subgraph $G[E(\mathcal{T})]$ which may not be a triangle-tree-structure, that is, it may contain a triangle-cycle-structure as a subgraph.

In $G$, for each $1 \leq i \leq c$, let $G_{i}=G\left[E\left(\mathcal{T}_{i}\right)\right], V_{i}=V\left(G_{i}\right), E_{i}=E\left(\mathcal{T}_{i}\right) ; E_{i}^{0}=$ $E\left(V_{i}\right) \cup \partial\left(V_{i}\right) \supseteq E_{i}$, and $G_{i}^{0}=G\left[E_{i}^{0}\right]$. We obtain a new graph $G^{\prime}$ from $G$ by doing Operation 1 at each edge $e \in E\left(V_{i}\right) \backslash E_{i}$ for $1 \leq i \leq c$, and we denote by $G_{i}^{\prime}$ the new subgraph (of $G^{\prime}$ ) corresponding to $G_{i}^{0}$. Applying Observation 2.3 repeatedly, we have $\operatorname{rc}(L(G)) \leq \operatorname{rc}\left(L\left(G^{\prime}\right)\right)$. Now each $G_{i}^{\prime}$ contains exactly $t_{i}$ triangles: $T_{i, j_{i}}$ where $1 \leq j_{i} \leq t_{i}$. We now obtain a new graph $G^{\prime \prime}$ from $G^{\prime}$ by doing Operation 2 to those $G_{i}^{\prime}$ s which contain triangle-cycle-structures such that each subgraph (of $G^{\prime \prime}$ ) $G_{i}^{\prime \prime}$ corresponding to $G_{i}^{\prime}$ contains no triangle-cycle-structure. Let $o p\left(G^{\prime}\right)$ be the minimum times of doing Operation 2 needed during above procedure. Clearly, op $\left(G^{\prime}\right)=$ $o p(G[E(\mathcal{T})])$ (minimum times of doing Operation 2 needed for $G[E(\mathcal{T})]$ such that the resulting graph contains no triangle-cycle-structure). Since there are op $\left(G^{\prime}\right)$ new inner vertices totally produced, and by Observation 2.4 and the discussion of Theorem 3.1, we have

Lemma 3.4 For any set $\mathcal{T}$ of $t$ edge-disjoint triangles of a connected graph $G$ with $n_{2}$ inner vertices, we have

$$
r c(L(G)) \leq n_{2}+o p(G[E(\mathcal{T})])-t .
$$

We know that a triangle-forest-structure of size $\ell$ contains $2 \ell+c$ (inner) vertices where $c$ is the number of components of it. Operation 2 does not change the number of edge-disjoint triangles, but the number of inner vertices increases 1 after we did Operation 2 once. Then it is easy to show that after doing Operation $2 o p(G[E(\mathcal{T})])$ times, the number of inner vertices of the new graph is op $(G[E(\mathcal{T})])+n_{2}=2 t+1+n_{2}^{\prime}$ where $n_{2}^{\prime}$ denotes the number of inner vertices not covered by the original $t$ edgedisjoint triangles. So, by Lemma 3.4, we have the following result and for the sharpness of the bound see Example 3.6.

Theorem 3.5 If $G$ is a connected graph, $\mathcal{T}$ is a set of $t$ edge-disjoint triangles that cover all but $n_{2}^{\prime}$ inner vertices of $G$ and $c$ is the number of components of the subgraph $G[E(\mathcal{T})]$, then

$$
r c(L(G)) \leq t+n_{2}^{\prime}+c .
$$

Moreover, the bound is sharp. 
Example 3.6 Let $G$ be a graph shown in Figure 3.6. The set $\mathcal{T}=\left\{u_{i}, v_{i}, u_{i+1}\right\}_{i=1}^{k-1}$ is a set of $k-1$ edge-disjoint triangles, $n_{2}^{\prime}=1$ and $c=1$. By Theorem 3.5 , we have $\operatorname{rc}(L(G)) \leq k+1$; on the other hand, it is easy to show that the diameter of $L(G)$ is $k+1$, and so $\operatorname{rc}(L(G))=k+1$.

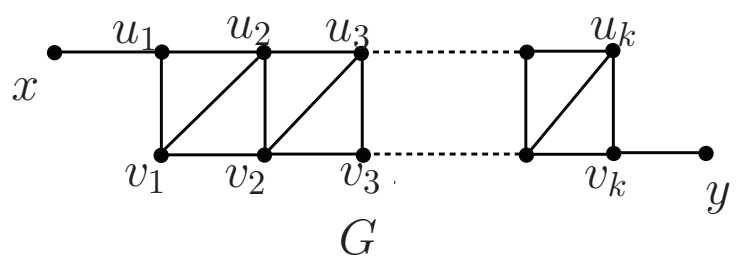

Figure 3.6 Figure of Example 3.6 .

\section{Bounds for iterated line graphs}

Recall that the iterated line graph of a graph $G$, denoted by $L^{2}(G)$, is the line graph of the graph $L(G)$. The following corollary deduced from Theorem 3.4 is an upper bound of the rainbow connection numbers of iterated line graphs of connected cubic graphs.

Corollary 4.1 If $G$ is a connected cubic graph with $n$ vertices, then

$$
r c\left(L^{2}(G)\right) \leq n+1
$$

Proof. Since $G$ is a connected cubic graph, each vertex is an inner vertex and the clique $\langle S(v)\rangle$ in $L(G)$ corresponding to each vertex $v$ is a triangle. We know that $\mathscr{K}=\left\{\langle S(v)\rangle: v \in \overline{V_{2}}\right\}=\{\langle S(v)\rangle: v \in V\}$ is a clique decomposition of $L(G)$. Let $\mathcal{T}=\mathscr{K}$. Then $\mathcal{T}$ is a set of $n$ edge-disjoint triangles that cover all vertices of $L(G)$ and $L(G)=L(G)[E(\mathcal{T})]$. So $n_{2}^{\prime}=0$ and $c=1$, and by Theorem 3.5, the conclusion holds.

In graph $G$, we call a path of length $k$ a pendent $k$-length path if one of its end vertex has degree 1 and all inner vertices has degree 2. By definition, a pendent $k$-length path contains a pendent $\ell$-length path $(1 \leq \ell \leq k)$. A pendent 1-length path is a pendent edge.

Theorem 4.2 Let $G$ be a connected graph with $m$ edges and $m_{1}$ pendent 2-length paths. Then $\operatorname{rc}\left(L^{2}(G)\right) \leq m-m_{1}$, the equality holds if and only if $G$ is a path of length at least 3.

Proof. Now $L(G)$ is a graph with $m$ vertices and $m_{1}$ pendent edges. Then it has $m-m_{1}$ inner vertices. By the discussion before Theorem 3.1, we give each 
clique $\langle S(v)\rangle$ in $L^{2}(G)$ a fresh color, where $v$ is an inner vertex of $L(G)$. It is easy to show that this gives a rainbow $\left(m-m_{1}\right)$-edge-coloring of $L^{2}(G)$, and so $r c\left(L^{2}(G)\right) \leq m-m_{1}$.

If $G$ is a path of length at least 3 , then the equality clearly holds.

If $G$ contains a cycle, then $L(G)$ clearly contains a minimal cycle $C: v_{1}, \cdots, v_{\ell}$. So $L^{2}(G)$ contains a clique-cycle-structure of size $\ell$ which is formed by cliques $\{\langle S(v)\rangle\}_{i=1}^{\ell}$. By a theorem in [6], we know that this structure needs at most $\left\lceil\frac{\ell+1}{2}\right\rceil$ colors to make sure that it is rainbow connected. The rest part of $L^{2}(G)$ is formed by cliques $\left\{\langle S(v)\rangle: v \in V^{2} \backslash\left\{v_{i}\right\}_{i=1}^{\ell}\right\}$ where $V^{2}$ is the set of inner vertices of $L(G)$. We give each $\langle S(v)\rangle$ a fresh color, by Observation 2.1, $r c\left(L^{2}(G) \leq\left\lceil\frac{\ell+1}{2}\right\rceil+\left(m-m_{1}-\ell\right)<\right.$ $m-m_{1}$.

If $G$ is a tree with a vertex of degree at least 3, then $L(G)$ contains a cycle, a similar argument will show $r c\left(L^{2}(G)\right)<m-m_{1}$.

So $G$ is a tree with maximum degree 2 , and hence it must be a path of length at least 3 .

\section{References}

[1] J.A. Bondy, U.S.R. Murty, Graph Theory, GTM 244, Springer, 2008.

[2] G. Chartrand, G.L. Johns, K.A. McKeon, P. Zhang, Rainbow connection in graphs, Math. Bohem. 133(2008), 85-98.

[3] Y. Caro, A. Lev, Y. Roditty, Z. Tuza, R. Yuster, On rainbow connection, Electron. J. Combin. 15 (2008), R57.

[4] S.T. Hedetniemi, P.J. Slater, Line graphs of triangleless graphs and iterated clique graphs, in Graph Theory and Applications, Lecture Notes in Mathematics 303 (ed. Y. Alavi et al.), Springer-Verlag, Berlin, Heidelberg, New York, 1972, pp. 139-147; MR49\#151.

[5] Bo-Jr Li, G.J. Chang, Clique coverings and partitions of line graphs, Discrete Math. 308(2008), 2075-2079.

[6] X. Li, Y.Sun, Rainbow connection numbers of line graphs, accepted for publication in Ars Combin. 